\title{
Hospital preparedness for COVID 19 pandemic, experience at base hospital Wellawaya, Sri Lanka
}

\author{
Nithin Ranawaka ${ }^{a^{*}}$, N.H. Welikumbura ${ }^{b}$ \\ a Postgraduate Institute of Medicine, University of Colombo, Sri Lanka \\ ${ }^{b}$ Postgraduate Institute of Medicine, University of Colombo, Sri Lanka \\ DOI: 10.29322/IJSRP.11.05.2021.p11343 \\ http://dx.doi.org/10.29322/IJSRP.11.05.2021.p11343
}

\begin{abstract}
COVID 19 is a global pandemic caused by newly discovered Corona virus. Hospital preparedness is essential to prevent spreading of infection to hospital staff, patients and provide good quality care to COVID 19 patient and other patients. This article shares the experience of hospital preparedness at base level hospital in Sri Lanka. Sharing experiences will help to learn lesson and enhance the capacity to face COVID-19 pandemic successfully.
\end{abstract}

Index Terms- COVID-19, Hospital preparedness, Hospital experience, preventive measures

\section{INTRODUCTION}

$\mathrm{C}$ orona Virus Disease-2019 (COVID-19) first reported in Wuhan, China, became a global pandemic. It is the $5^{\text {th }}$ documented pandemic since the 1918 flu pandemic (1). The first Sri Lankan local COVID 19 positive case has identified in March 2020 (2). Since then, in Sri Lanka, more than 100000 COVID 19 positive patients identified and more than 500 deaths were reported(3).

In Sri Lanka, some hospital has converted to COVID-19 treatment centers that facilitate treatment for COVID 19 positive cases. When other hospitals isolate COVID 19 positive patients, immediately transferred to the COVID treatment centers.

According to the ministry of Health, nutrition and indigenous medicine Sri Lanka, there are several categories of hospitals, such as National hospitals, Teaching hospitals, Provincial General Hospitals, District general Hospitals, Base Hospitals (A or B), and Divisional Hospitals (A, B, C). Base Hospital Wellawaya (BHW) is a B grade Base Hospital situated in Monaragala District. Bed capacity is 122. This hospital functioning as a non-COVID center. BHW supervised by the Regional Directorate of Health Services Monaragala and the Provincial Directorate of Health Services Uva province. Currently, there are 29 medical officers, (including Medical Superintendent, two consultant physicians, and consultant pediatrician), 68 Nursing officers (including special grade nursing officer, two sisters, and master), 10 office staff (including administrative officer) working in hospitals. Basic facilities like $\mathrm{x}$ ray, ECG, biochemical analysis, and hormonal assay available in hospital. BHW does not have a theater complex, premature baby unit, or Intensive care unit.

Ministry of Health (MoH) Sri Lanka issued several guild lines, circulars on how to prepare a hospital for COVID 19 pandemic situation. Based on those, BHW has prepared its own preparedness plan to face the COVID pandemic.

\section{1) Effective leadership}

Hospital COVID management leadership taken by COVID cell. It has 15 members including Medical Superintend, all consultants, Medical officer in charge of outpatient department, administrative officer, matron, infection control nursing officer, chief pharmacist, and chief medical laboratory technologist. COVID cell meet whenever the need arises and weekly. All the important and main decisions on a response to COVID 19, taken by COVID cell.

Subcommittee has appointed to get decisions on isolation or quarantine the staff of hospital after risk assessment. Medical superintendent of the hospital, Consultant physician, Consultant pediatrician, Matron and infection control nursing officer appointed for subcommittee. Whenever information receives regarding contact of COVID 19 patient with hospital staff, the following steps will take.

a) Confirm the information

Information may receive from a representative from the Medical officer of health in Wellawaya area, a representative from the regional directorate of health Monaragala, by hospital staff, or by the contacted person.

b) Collect all necessary information

Information will collect by the infection control nursing officer via telephone or by any other member of the subcommittee. Hospital has prepared separate forms to fill details on risk assessment according to the circular issued by MoH Sri Lanka. After collecting information, subcommittee members arrange meeting physically or via group call. After the discussion, members will come to a conclusion (whether high, intermediate, or low-risk exposure, whether to quarantine or not) about the individual who exposes to COVID 19 patient.

\section{2) Infrastructure preparedness}

a) Isolation unit for COVID suspicion patients

There was an underutilized old two-story building used for clinics. According to the decision of COVID cell in hospital, clinics have shifted to another building and cleared 4 rooms. Those 4 rooms ( 8 beds) have utilized for isolate COVID 19 suspected patients. Another room arrange for staff and front porch utilized for emergency management. Necessary instruments and beds mobilized from other units of the hospital. One male nursing officer appointed as in charge of the unit. 
b) Triage areas

Three triage areas have prepared by using temporary huts.

c) Isolation/quarantine area for hospital health staff.

There was a necessity to arrange an area for isolation/quarantine health staff, who have exposed to COVID 19 suspected or positive patient while on duty. There was an underconstruction building in hospital and one floor of that building has prepared for isolate/quarantine hospital staff. Area separated and covered by transparent polythene sheets.

3) Gate keeping, triaging, outpatient, and emergency patient management.

BHW have three gates. One gate allocated for people who come for the outpatient department (OPD), one gate for the emergency patients. Third gate for visitors and clinic patients.

\section{Gate 1}

Gate one reserved for OPD patients. At the gate entrance, a sink with a water supply has fixed for wash hands. A security officer appointed near the gate to check temperature of the entering patients. The temporary hut has built and covered by transparent polythene and used as the first triage area. One nursing officer appointed there for initial triage. Triage will do as follows COVID 19 suspected - patient direct to an isolation unit, prepared for COVID 19 suspected patient.

If need medical officers' opinion or further evaluation Patient directed to the second triage point with video, audio facility to discuss with medical officer in OPD. Medical officer will decide whether give treatment in OPD set up and send home or whether to direct isolation unit or to admit casual wards.

No COVID 19 suspicion - patient directed to medical officer OPD for treatment.

Gate 2

Gate 2 reserved for emergency patients. In here patients directly go to the OPD building front porch. Front porch equipped with basic facilities to handle emergencies. One nursing officer appointed to front porch with full personal protective equipment. After initial assessment and according to the history, if there is a suspicion of COVID 19, the patient will transfer to the isolation unit emergency treatment area after stabilization. If the patient not COVID suspicion patient, he or she will admit to a casual emergency treatment unit.

Gate 3

Gate 3 reserved for clinic patients and for visitors. Security officer at the gate will write all necessary information about the visitors.

Only one visitor allowed to visit one inward patient per day. Nursing officers take information about visitors from inward patients and acknowledge the security officer at gate.

In the clinic days, one nursing officer appointed at gate 3 to triage the clinic patients.

4) Clinics management
Crowd is the main challenge in the clinics. Patients who do not have to consult regularly by medical officers, have selected and their clinic drugs sent by post to their home monthly. For other patients, pre-decided time have given for consultation in clinic days. All clinic patients triage before entering to the clinic building by nursing officer. A separate telephone number has preserved for clinic booking.

5) Droplet precautions, personal protective equipment (PPE), social distancing

Regular training for donning/doffing was conducted to ensure health care workers were fully conversant in PPE by the infection control nursing officer. PPE provided to health staff according to the guideline of MoH Sri Lanka. Maintain buffer stock of PPE and level of PPE balanced weekly. Frequent lectures on COVID 19 and rational use of PPE has conducted by a Consultant physician and Medical Superintendent.

6) PCR testing

Daily 5 random PCR done in hospital. Weekly 5 random PCR test done for health staff of the hospital.

\section{CONCLUSION}

Base hospital Wellawaya took number of drastic control and preventive measures with minimal resources and able to face this pandemic successfully up to now. All activities related to outbreak response should continue with regular review of the process and making of necessary adjustments until this pandemic eliminated globally.

\section{REFERENCES}

[1] Liu YC, Kuo RL, Shih SR. COVID-19: The first documented coronavirus pandemic in history. Biomed J [Internet]. 2020;43(4):328-33. Available from: https://doi.org/10.1016/j.bj.2020.04.007

[2] Nanayakkara S, Nakandala P, Nakandala U, Nanayakkara I. A Review on COVID-19: A Global and Sri Lanka Perspective. Am J Multidiscip Res Dev (AJMRD [Internet]. 2020;2(8):63-70. Available from: https://orcid.org/0000-0001-7832-9621

[3] Ministry of Health. covid-19-Hospital preparedness. 2020.

\section{AUTHORS}

First Author - Dr. Nithin Ranawaka, M.D. (Kursk State Medical University), PG Diploma in Tuberculosis and Chest Disease (Postgraduate Institute of Medicine, University of Colombo), MSc in Medical Administration (Postgraduate Institute of Medicine, University of Colombo)

Institute - Postgraduate Institute of Medicine, University of

Colombo, Email - ranawakanithin@yahoo.com

Second Author - Dr. Nisansala Hemakumari Welikumbura PGM (Kursk State Medical University), Institute - Postgraduate Institute of Medicine, University of Colombo 